\title{
Hermeneutics and the Principle of Explicability
}

\author{
Ted Toadvine \\ University of Memphis
}

In Interpretation and Explanation in the Human Sciences, 1 David Henderson unveils a proposal to displace previous conceptions of the interpretive enterprise with a holistic scheme encompassing the previously accepted "Principle of Charity," while elegantly explaining its limitations. Not to stop short, Henderson goes on to illustrate the implications of his interpretive codification, branded the "Principle of Explicability," for attributions of irrationality, and, more importantly, for the relation between the method of the human sciences and the natural sciences. As we shall presently show, and Henderson himself is aware, there are explicit similarities between his account of interpretation and accounts offered in the hermeneutic tradition, particularly with regards to the interpretive constraint of holism. However, the two traditions focus on different questions and problems. Henderson is concerned with issues stemming from the problem of "radical translation," characteristic of his Anglo-American predecessors Quine and Davidson; Hans-Georg Gadamer, of the Continental school of hermeneutics, focuses on the issues involved in understanding a text, particularly that of another culture and time. While some differences in method will stem from differences of available evidence, the question with which we are here concerned is this: Are basic concepts employed in the interpretive account presented by Henderson the same concepts employed by Gadamer? What we intend to show is that the "Principle of Explicability" does have applicability within the interpretive practices described in Gadamer's "On the Circle of Understanding,"2 demonstrating a link between the interpretive approaches of the Anglo-American and Continental Traditions. Differences in emphasis, however, point out the subtle variations between the accounts, contrasts which may raise questions about their further resolution. We will begin by detailing Henderson's account, turning then to Gadamer in order to relate salient themes.

\section{Henderson's Principle of Explicability}

Henderson's work on interpretation ${ }^{3}$ takes place against a background of fairly unanimous agreement by other theorists on two basic counts. The

I David Henderson. (1993). Interpretation and Explanation in the Hunan Sciences. Albany: SUNY Press.

2 Hans-Georg Gadamer. (1988). "On the Circle of Understanding." In J.M. Connolly and T. Keutner, trans. \& eds., Hermeneutics versus Science? Notre Dame: University of Notre Dame Press, pp. 68-78.

3 Although Henderson finds it helpful to distinguish between translation schemes (applying to language) and interpretive schemes (applying to behaviors) (p. 15), here 
first of these, that interpretation must be holistic, is represented by the notion of the "hermeneutic circle":

[W] hat interpretation is proper for a particular bit of text or behavior is dependent on what interpretation can be settled on for the larger text or range of the agent's behavior, of which the particular bit is a part (p. 3 ).

Accordingly, no meaning or explanation for a bit of text or action is possible without taking into account the surrounding context of words and behaviors. In practice, this amounts to a back-and-forth reading of the whole against the parts, slightly altering the larger picture as the necessity of making sense of the parts suggests, and similarly re-reading the parts to realize a sensible whole. In terms of understanding behavior, the anthropologist is required to account for the actions of a foreign culture by seeing individual acts within the context of the behavior of the culture as a whole. To give an everyday example, imagine explaining the simple action of "stopping at a traffic light" to someone radically unfamiliar with our culture: such an explanation would make sense only within the larger explained context of automobiles, roads, intersections, and traffic laws, at the very least.

Extreme holism, of the sort which insists that no individual action makes sense without a complete understanding of the whole, seems less well-grounded. Understanding traffic-lights requires familiarity with a certain context, but probably not explicit knowledge of, say, fast-food restaurants. The two items, both quite possibly unfamiliar to mentbers of certain cultures, have contexts which overlap, but are not particularly closely related. Thus an explanation of either could plausibly be couched in terms not mentioning the other. Generally, the size and extent of the context necessary for explanation will be dependent on the strength of the relations between the areas being explained.

Further, holism prescribes that an interpretation of actions must go hand-in-hand with an interpretation of linguistic meaning. This is simply because "utterances and (other) actions are so intimately associated that if we could not sort out what a people were doing, we should not think that we understood what they were saying" (p. 16). On the other hand, discrimination with regards to the meanings of behaviors relies on an understanding of the utterances accompanying them. With regard to holism, then, Henderson accepts the basic view that explanation situate itself within the Zusammenhang of actions and utterances to be explained,

we will be speaking broadly of all interpretive practices as "interpretation." Citations within Pan l of this essay will be from Henderson (1993), unless otherwise identified. 
while rejecting the stronger claim that explanation of any action would require complete understanding of its interconnections with the whole. 4

The second count of agreement by contemporary theorists is that interpretation is constrained by a methodologically-fundamental "charity." Typically, this is construed to mean that interpreters necessarily translate words or actions in a manner most consistent with normative rationality, as any evidence within a translation of irrationality would count strongly against the acceptability of the translation. This is to say, a translation's best evidence for its own success is its ability to successfully construe its subjects as (mostly) perfectly consistent by the norms of logic. Henderson identifies this as the "Standard Conception" of charity:

The Standard Conception of charity in interpretation: we are bound by a fundamental methodological constraint to find people rational (p. 33).

It is important to realize that this conception makes two fundamental claims, both of which Henderson intends to overturn: (a) charity is a "fundamental methodological constraint" rather than derivative of a more fundamental codification of interpretive practice, and (b) charity thus binds interpreters to find their subjects rational.

The obvious problem with the Standard Conception is that, "[s]ince, as a matter of fact, no interpretive scheme ever makes people out to be fully rational belicvers of only true things, no interpretive scheme is ever really adcquate" (p. 34). We are, in effect, bound to continually adjust our interpretive scheme until all subjects are perfectly rational. Since this seems rather at odds with actual interpretive practice, certain limitations to the Standard Conception have been devised. The two most important attempts, which Henderson discusses in detail, 5 are (a) to modify the application of charity according to the stage of interpretation, and (b) to modify interpretive charitability based on the relative "rational value" of different sorts of errors. Henderson is convinced, however, that such ad hoc modifications to the principle of charity point out the necessity of a more fundamental codification for interpretation.

This more fundamental principle, christened the "principle of explicability," represents the genuinely essential concerns of "social scientific endeavor: studying and explaining similarities and variations in beliefs and practices" (p. 51). What is intended by making "explicability" fundamental is simply to emphasize that the primary concern of the interpreter is to explain behaviors or texts. Although charity has its role, which is indeed a necessary one, charitable attributions are merely a

4 For a comprehensive discussion of these issues and their roots in the work of other theorists, see Henderson (1993), pp. 14-26.

5 See Henderson (1993) pp. 36-49. For a more thorough discussion of the Principle of Charity and Henderson's formulation of its Standard Conception, see pp. 26-36. 
stepping-stone to later stages of interpretation, and thus more advanced explanation.

In the initial stages of interpretation, a "first approximation scheme" is formed from available evidence and charitable contributions. At this stage, charity is necessary. When I know none of an interlocutor's tongue and am struggling to match words and phrases with bits of the environment, I can only assume she or he is candidly responding to my queries with genuine assent or dissent, and that she or he is basically consistent and rational. Finding errors of unexpected irrationality at this stage would indeed be evidence of poor translation, simply because translation has no other option but to maximize agreement. 6 But even in this stage, the operative principle is explication, at least potentially, of the interpreted behaviors. Establishing this first level of agreement sets the stage for the possibility of "genuine" (uncharitable) agreement or disagreement. A first-approximation scheme is necessary for these reasons:

(1) only by using such a scheme can the identification of putative error be made sufficiently precise and well-grounded to warrant investigation, and (2) well-grounded information needed for the investigation of such attributions of error for explicability is accessible only through the use of such a scheme (p. 52).

Thus the ultimate purpose of charity is seen in the construction of a firstapproximation scheme for interpretation, the purpose of which is finally explication.

With such a scheme in place, error will certainly rear its head; this shouldn't however force us to question our scheme, but rather to seek explanation for the error. What counts as error needn't necessarily be poor reasoning or surprisingly incorrect beliefs; unusually sharp reasoning skills in the untrained or surprisingly advanced beliefs in technologically primitive societies can just as possibly appear anomalous. This is simply because normative considerations do not determine the structure of our interpretive scheme. With explicability as our guide, what we will most reasonably read into another's actions will be what is most expected, and thus most explainable. In other words, the constraint to charitably prescribe rationality according to our norms gives way to the prescription of rationality or irrationality according to our best descriptive generalizations. Such "errors of rationality" as arise by these means, which are only such from the perspective of developed logical norms or science, may be

6 Agreement is maximized, but not based on normative considerations. Rather, weighting to the Principle of Charity will naturally occur with regards to certain sorts of errors due to the appropriate descriptive generalizations concerning where errors are most likely (as will be explained below). Thus the earlier attempts to limit charity with regard to sorts of errors correctly reflected practice but was incorrectly understood. 
obviously explicable. In the case where such errors are not yet explicable, due to the limitations of our current theory or information, but are in kecping with our descriptive generalizations, they remain potentially explicable when our theory develops sufficiently or more information is gathered.

But other errors do arise after the first-approximation scheme is sufficiently developed to provide a background of non-anomalous behaviors against which they may appear. Again, even errors which go against our earlier descriptive generalizations are not to be discounted; they must simply await explication at a later stage of our theory's development or at the gathering of more information. Only those errors which defy explicability even at a late stage in the development of our scheme may cause us to look back at some of the initial assumptions of our firstapproximations. The stage at which any inexplicable errors may appear must already be one which has advanced to the stage of holism, of sufficiently broad context to allow errors in some areas to appear at odds with beliefs in others.

Obviously, at the stage where genuine errors appear, we have left charitability behind. Thus, the earlier attempt to modify the Principle of Charity with regards to stages of interpretation brought to light an important aspect of the interpretive process only appreciable when charity has been subsumed under explicability:

With access to generally correct information regarding the heliefs of source-language speakers, the time has come to get on with the central social-scientific business: explanation. Accordingly, the constraints of the principle of charity grade off and eventually dissipatc entirely in the later stages of interpretation....[W]e recognize that the principle of explicability...guides the investigator in the later going (pp. 556).

In the later stages of interpretation, the emphasis is on the explicability of the anomalous beliefs unexplained until that point; charity is no longer at issue. Thus, the Principle of Explicability subsumes even those stages in the interpretive process previously outside the range of the Principle of Charity. We see, then, that "[I]nterpretation is not a matter of imposing a normatively derived structure on those interpreted, rather, it is a matter of theory-laden description of cognitive and social phenomena" (p. 60).

Two points about the claim that explication is "theory-laden description of cognitive and social phenomena" need to be made. The first is that the proper explanatory value of such description is realized in terms of its ability to model "relevance relations." Since the description which is interpretation is of social phenomena, it must capture a set of relations which obtain between dispositions for behaviors (including dispositions for verbal behaviors) for the subject social group. Thus, dispositions within the 
normal social context will be related to other dispositions in ways which may be modeled. For instance, dispositions to assent to a certain sentence may imply dispositions to assent to a second, under certain conditions and for certain reasons. To correctly model these relations in English, we would need to translate the two sentences in such a way that they maintain the same relationship (for speakers involved in a belief system of the sort described). Establishing these relations is more complex than simply recognizing normative relations between certain dispositions, which leads to the second point.

As "theory-laden descriptions of cognitive and social phenomena," our modeling must invoke our theoretical understanding of cognitive processes and social phenomenon. This is to say, interpretation must be seen as the "task of interpretive schemes and psychological (and sociological) theory (together comprising a global theory), not the task of translation or interpretive schemes alone" (p. 71). The point goes back to our earlier mention that the construction of an interpretive scheme is dependent on generalized descriptions. These descriptions are characterizations of the way that people behave, the way they think, and the way they deviate from normative rationality. Construction of a first-approximation interpretive scheme must be heavily reliant on current psychological theory for its expectations, i.e. to weight the charitable appropriations. Explanation at later stages is basically the application of appropriate theories about peoples' behaviors in the interest of explaining instances of such behaviors. Resultantly, the testing of an interpretive scheme, applying the model which it constructs, is the testing not only of the scheme itself but of the embedded psychological and sociological theories as well; the failure of the model may indicate a lack in either component.

It should be clear from the explanation given here that the Principle of Explicability solves the problem of attributions of irrationality which caused the Principle of Charity such trouble. Since we are constrained not to find our subjects preponderantly rational but to find them minimally predictable via our previous descriptive generalizations about behavior, neither rationality nor irrationality are implicitly explainable. Thus, irrational behaviors are as potentially explicable as rational behaviors, or more so in certain situations. If, for instance, my experiences with Southern Baptists suggest that, in the face of explanations concerning the Paradox of Omnipotence, they continue to assert that God is omnipotent while at the same time admitting the paradoxical nature of this claim, this error may become part of a descriptive generalization (at the level of folk psychology at least; more accurate and precise empirical study may lind a link which goes beyond the folk-psychological level, of course). Forming a descriptive generalization is not, in itself, suitable explanation, however; what is necessary is for the particular occiasion of the act described to qualify as an instance which fits with our generalization, and therefore be explained through the theories which count toward explaining the generalization itself. 
In the case of the persistent Baptist, psychological and sociological theories will shed light on the tenacious nature of religious convictions, answering our questions of both how and why such behavior is brought about within the systematic relations of the subject's beliefs.

In response to the question of whether such a global theory as the one we are suggesting would be self-validating, Henderson points out that theories constructed according to such practice have not eliminated anomalies (p. 75). Nor can they be expected to do so until we have at our disposal

a set of psychological and sociological theories that can be combined with interpretive schemes for all people, and when so combined, lead us to model and expect what we do observe. This, like any ideal of empirical adequacy for scientific theory, is a high goal and not easily attained (p. 76).

We are not merely forcing anomalies into conceptual boxes to explain them away. Rather, like any scientific thcory, interpretation progresses through continual upgrading of the theoretical implements of which it makes use, approaching the point where current anomalies can be explained via correct theories.

We see here the connection Henderson wishes to draw between the sort of explanation appropriate in the natural sciences and that of interpretive procedures within the human sciences. Previous acceptance of the Principle of Charity as "a fundamental methodological constraint on adequate interpretation...makc[s] for a deep difference between interpretation and the description of phenomena in the other sciences" (p. 4). Given the Principle of Explicability, and the way by which it situates interpretation within a global theory drawing on sources from other scientific areas, we see that it advocates

the epistemically sound strategy of seeking to fit what is observed within our best descriptive understanding of "how the world is," particularly with respect to the cognitive capacities and social processes of the creatures under study....The principles governing when and where to make adjustments are...familiar, for they are the same as those governing theory building, testing, and application in all scientific domains: simplicity of scheme, relative confirmation of relevant bits of theory, the relative promise of lines of research, and so forth (p. 250).

Consequently, the claim of methodological separatism collapses with the recognition that the Principle of Explicability aptly presents the bona fide codification of actual interpretive practices.

In bricf summary, Henderson's Principle of Explicability leads us to answer positively the question of whether interpretation gives us an 
adequate means of comparing our views with those of another culture. We are not bound by methodological charity to merely read off what we have written into the translation manual, finding our subjects perfectly rational according to our norms. In striving to account for the views of others, we are bound to begin charitably, establishing a certain framework of correspondence between our views and those foreign to us. But this charity is in the service of bringing us to the point where the genuine interpretive work begins: where do our subjects surprise us with startlingly accurate or inaccurate views, and what reasons can we give for these anomalies? Working with a global theory, composed of dove-tailed research about the way we believe the world to be, we set out to find the areas where others don't meet our expectations. Ultimately the goal of such interpretive inquiry is to arrive at an understanding of the way things are, particularly in the areas of psychology and sociology, which will allow us to explain our differences from others, thereby understanding the relevant relations obtaining between their behaviors and beliefs. This explication, answering the hows and whys of others' behaviors, is the guiding principle for correct interpretive practice, and shares its basic methodology with the practices of the natural sciences. Let us turn now to Gadamer's approach, keeping the important issues from this theory in mind.

\section{Gadamer and the "Circle of Understanding"}

The basic insights of Gadamer's theory of interpretation are drawn from the concept of the "hermeneutic circle," mentioned above in the context of holism. For Gadamer, the holistic aspect of the process of understanding is aptly represented by the notion of the interpretive circle, but other aspects are represented therein as well: interpretation proceeds by way of approaching a text or action with certain expectations about the state of affairs the text describes. This is to say, the first stage of interpretation is one in which our understanding of the world is read into the subject of our study by a sort of anticipatory prejudice. The failure of the text to equal our expectations opens the possibility of the undermining of our prejudices; by failing to say about reality what we had expected, the text brings our presuppositions into relief. At this stage, the possibility is opened for a matching of our network of beliel's with those expressed within the text, and differences show themselves as disagreements about the object of discussion. Valid interpretation strives to allow the opening of this possibility by situating itself between an objective view of the world and the view offered by the tradition from which the text emerges. Our discussion here will be directed to clarifying these issues as they are articulated in "On the Circle of Understanding," with the aim of relating this approach to the account previously examined.

The rule of the hermeneutic circle is familiarly described as the necessity of understanding the whole in terms of the parts, while 
recognizing that the parts can only be given meaning under the projection of meaning for the whole. All interpretive procedures, all attempts at understanding, fall under the domain of this rule, as they strive to give meaning to contextually determined and connected actions and words, both spoken and written. "The anticipation of meaning, in which the whole is projected, is brought to explicit comprehension in that the parts, determined by the whole, determine this whole as well" (p. 68). ${ }^{7}$ That Gadamer's emphasis here is not simply that interpretive procedures must be holistic is apparent in his mention of meaning as "projected." His example is of the experience of learning a foreign language. In order to embark on an inquiry within the circle, one must make an initial projection of meaning to the whole; thus one gives a general idea of the meaning of the text to the whole in the translation of the first sentence. This overall meaning is clarified or rejected as more sentences are given a reading, and in the event of rejection, we return to the first sentence again, modifying our translation according to our new expectations. Thus, we see a back-and-forth movement between the reading of the whole and the part; this is the holistic aspect of the circular method. But we also notice the need for initial projections of meaning; there can be no opening to the circle without expectations found in the interpreter rather than the text.

Surveying the history of the hermeneutic tradition, Gadamer critiques the previous notion that understanding another's speech or writings requires getling inside "the author's inner state; rather, if one wants to speak of 'placing oneself, we place ourselves in his point of view....[W]e try to let stand the claim to correctness of what the other person says" (p. 69). This is to say, we recognize that the other person says something about something; a claim is being made about the world, and our job is to relate ourselves to this claim, not to the intentional statc of its author. Clearly, the author's subjectivity can provide little illumination in the case of understanding ancient texts. But how do we go about "letting stand" the other's claim, and how do we relate ourselves to the other's point of view?

For clarification, Gadamer turns to a passage from Heidegger's Being and Time:

[Interpretation's] first, permanent, and final task remains that of not accepting from flashes of inspiration and popular notions a pretence of its own fore-having, fore-sight, and fore-conception, but rather to work these out of the subject matter itsclf and thereby to sccure the topic under study. 8

7 Citations within Part II will be from Gadamer (1988), unless otherwise identified.

8 Heidegger, M. (1927/1962). Sein und Zeit. Tübingen: Verlag. Trans. as Being and Time by J. Macquarrie and E. Robinson. San Francisco: Harper \& Row, p. 153/195. The translation offered here is that provided by Connolly and Keutner in their translation of Gadamer, p. 70-1. For Heidegger's own explanation of and distinction 
We can equate the notion of "fore-conception" with the expectations brought to the text by the interpreter in our example. Heidegger's directive is that we separate out the appropriate expectations, those worked "out of the subject matter itself" and true to the "topic under study," from the prejudices we have picked from the "popular notions" around us. In other words, certain views are true to the world and may be gained through appropriate study; correct interpretation requires that we approach the text with the expectations that it accord with these true-to-the-world views, rather than to ungrounded expectations gained from our biased cultural training or elsewhere.

We shouldn't be misled into thinking that the suggestion here is to throw off our projections and just see the text "as it is." Interpretation can only begin with a projection of meaning onto a text, seeing it as a coherent unity which embodies some particular view of the world. It is this expectation of a definite meaning which allows the attribution of tentative meanings and continual revision as the text is unraveled. But "it makes good sense for the interpreter, animated by his ready pre-opinion, not to tackle the 'text' straight off, but rather to test the living pre-opinion in himself for its legitimacy, i.e. for its provenance and validity" (p. 72). In other words, the object under discussion in interpretation of a text is (at least initially) less the text itself than what the text is about. If one is to understand a text, one must first align her or his understanding of the world, since understanding the text requires "setting it in relation to the whole of one's own opinions" (p. 72).

Awareness of our own pre-conceptions allows the shift from reading our expectations into the text to reading the text as an alternative view of reality. The valid interpreter is not interested in merely discovering her or his own views reflected back from the text because they have been read into it; rather, knowledge about her or his preconceptions allows the strangeness of the text to reveal itself, giving the text "a chance to play off its truth in the matter at hand against the interpreter's pre-opinion" (p. 73). According to Gadamer, who follows Heidegger's work on this point, the break between one's own attributions to the text through pre-opinionated expectation and the view implicit in the text itself is discovered through the interpreter's "historical consciousness" (p. 74). Put simply, the interpreter must be explicitly aware of the prejudices with which he or she approaches the texl, recognizing their origin, implications, and historical situatedness. To give an example of this method in practice, consider the contemporary reader of an English translation of Genesis. Comprehending the biblical references to the "firmament" requires knowledge about ancient cosmological beliets; how these beliefs and their implications will be understood by us depends on our own knowledge of the universe, including the implications our

between fore-having, fore-sight, and fore-conception, see Heidegger (1927/1962), p. $150 / 191$. 
bcliefs have for us and the different origins and methods from which ancient and contemporary theories about such matters are derived. Hence, knowledge of the historical situation of our own knowledge provides a grounding against which competing theories of other eras can be explicated.

Returning to the first movement of our hermeneutic circling, the proper initial expectation, according to Gadamer, is "the anticipation of perfection:"

It says that one can only understand that which represents a perfect unity of meaning. For example, we make this presupposition of perfection whenever we read a text. We only call this presupposition into question if it proves irredeemable, i.e., the text does not become comprehensible; perhaps we begin to have doubts about the authenticity of the text and set out to confirm it....The anticipation of perfection which guides all our understanding thus turns out to be one determined in each case by content...[T]he reader's comprehension is also constantly guided by transcendent expectations of meaning which arise from the relationship to the truth of what is meant (p. 74).

We anticipate perfection in the text on two counts: unity and truth of reference. The first, as we have seen, is the presumption that the putative unity of the text's meaning will become apparent as our interpretation narrows in through its back-and-forth adjustment of meanings of parts and the whole. The second, that the text's statement about the world will turn out to be correct, is the upshot of the necessity of our reading into the text our entire view of the world (or, in a particular case, certain aspects of our global understanding). It is important to notice that what is naturally and immediately imputed to the text is the interpreter's own primary beliefs, rather than the beliefs which he or she may "know" to be correct. This is to say, rather than using technically correct information about the world, the interpreter will tend to read the subject text as incorporating the knowledge closest to him or her; perhaps if the text is making technical scientific claims, that realm of knowledge would come into play, but, generally speaking, the subject will be understood to articulate views only as technical as the interpreter's normal "folk" views.

The breakdown of this charitable scheme comes when enough information has been gathered to realize the text fails, and we cannot continue to construc it as unified or correct by our normal standards. This is the point, at least with regard to the latter issue, where attempts to "explain" the text become important. "It is only the failure of the altempt to admit what is said as true that leads to the endeavor to 'understand' -psychologically or historically - the text as the opinion of another" (p. 75). My interpretive emphasis shifts at this point from what is being said to the strangeness of its way of saying, requiring my invocation of 
psychological or historical explanation to account for the error on the other's part.

But the error may not rest with the other; it may rest with my own understanding of the world. It is also at the point where disagreements arise that my own prejudices are called into question. The opposing view presented by the text demands that I re-examine my expectations; are they demanding that the text conform to a view of the world which is incorrect, and can the text present a view which converts me? As Gadamer makes clear, interpretation is often thought to be hardest with respect to those writings or actions which come to us from the distant past. But, on this count at least, the trials of time and the history of interpretation of these writings and events comes to our aid:

Filtering out the true meaning contained in a text or an artistic creation is, incidentally, itself an unending process. The temporal distance which accomplishes this filtering is engaged in a constant movement and enlargement, and this is the productive side which it possesses for understanding. It lets prejudices which catch only a part of the work die off, while letting those emerge which make possible a true understanding (p. 77).

We may stand so close to our own prejudices, embodied within the culture to which we are socialized, that the false bias these opinions give us misses the meaning the text articulates. Through time, interpretations linked to the biases of a particular culture and era die out, allowing the true meaning of the text to gradually come to light.

But the help of a history of interpretation for a text is not always available, and we must, therefore, cultivate the necessary historical consciousness to recognize our own biases. In effect, this process occurs along with interpretation, as our implicit opinions come to light against the contrast created by meanings within the text. With our biases made apparent in this manner, we can suspend judgement about the state of things in question, allowing for the possibility of our own error and the possibility of learning from the text:

The truth is that one's own prejudice only really gets involved in the game by becoming itself at stake in the game. Only by playing out its role can it become so teamed up with the other that it too lthe other] can play out its role (p. 78).

Therefore, the alien aspects of the text under consideration can only be appreciated as alien, as radically differentiated from my own beliel's, when my expectations are foiled, and the differences between my view of the world and that articulated by the text are given room to emerge. 
The emphasis in Gadamer's account is ultimately on the interplay of opinions and prejudices between the interpreter and the subject of interpretation. Both bring to their meeting point a perspective on the nature of the world mediated by a tradition. The job of the interpreter is to stand somewhere between the tradition put forward in the text and the truth of the matter in question: close enough to the text to allow its view to emerge, but far enough away to hold this view against his or her own expectations, which are really the whole of his or her opinions about the world. Initially, we must enter the holistic interplay of interpretation by expecting the text's views to match our own, and expecting a unity of meaning to unfold between the parts and whole. If the unity of meaning we seek can't be reconciled with our reading, or the views don't match our expectations when it unfolds, we enter the task of explanation: we set the texi's claims about the world against ours, and if ours hold, we relate the viewpoints of the lext's tradition to our own to find reasons for the divergence. If, however, our own incorrect biases come to light, our examination of them provides us with an opportunity to learn about the world from the text. The explanatory task turns toward realizing the origins of our own false biases. With this characterization in place, we can now turn to the task of relating Henderson's and Gadamer's accounts.

\section{Explication as a Moment of the Hermeneutic Circle}

From the explanation of these two accounts, several points should be clear. First, the basic emphasis on charity in the initial stages of interpretation is similar. Approaching the subject with a set of expectations gives the interpreter the opportunity to achieve a basic bridgchead of similarities with which to work. This charitable approach is weighted in either case, although we will need to examine the differences between the accounts of this weighting. After the initial stage has provided this similar background, the possibility of disagreement (or genuine agreement) emerges, and the actual interpretive work gets underway. Secondly, contrasts emerge. Henderson thinks of this latter stage in terms of the explanations we will need to give for surprises; why haven't the natives matched our descriptive generalizations? Accounting for this requires continued study of the way the world operates, particularly with regard to psychology and sociology, in the hopes that our theories will turn up explanations for what seem to be anomalous behaviors. Gadamer, whose work is directed more toward the study of texts, focuses less on our need to explain surprises to our expectations than on the possibility of our learning from the text. This difference arises from a contrasting focus, but may lead us to important insights into each account. Here our goal will be to bring the implications of each theory into the open to see the questions each raises about the other. 
Henderson suggests that certain charitable contributions be made to get the study underway. We need to take the natives' utterances and their assents and dissents at face-value; we need to assume that they are making meaningful statements, which can eventually be understood; and we need to approach them with expectations derived from our own sociological and psychological observations and generalizations. About the first two claims, Gadamer is in accord: we read the text with expectations of a coherent meaning, assuming that it has something to say about something. But on the latter point, concerning the informing of our expectations about the world, he takes a slightly different approach:

Just as the addressee of a letter understands the news he receives
and, to begin with, sees things with the eyes of the letter writer,
i.e. takes what the writer says to be true - instead of, say, trying
to understand the writer's opinion as such - so we too
understand the texts which are handed down on the basis of
expectations of meaning drawn from our own relationship to the
issues under discussion (Gadamer, pp. 74-5).

This point relates directly to how the principle of charity in early stages of interpretation should be weighted. The contrast is a subtle one: Henderson sees our weighted principle of charity as an application of descriptive generalizations of current psychological and sociological theory. In the case of a recently-trained anthropologist, we may indeed expect the charitable approach to be influenced by such recent and highly-theoretical scientific endeavors. But consider "normal" everyday communication: the typical student faced with ancient philosophy or an American tourist in a foreign land. The expectations in these cases will be determined by "our own relationship to the issues under discussion." This difference in account could merely reflect the difference in focus between the two theories. The following two questions, however, are raised: (a) is Henderson's account a codification of actual interpretive practices or of a normative guide for such practices, and (b) granting that our psychological and sociological theories give us information about the way people process information, are we not ourselves therefore subject to the failings of normative rationality explained therein?9 These questions raise issues beyond the scope of this paper, but we may want to ask if our recently trained anthropologist is different in kind from the American tourist, or has she simply embodied a radically different relationship of her own to the "issues under discussion."

9 To put this more clearly, our psychological theories tell us that people do not think according to normative rules. In our own application of descriptive generalizations within a charitable attribution, therelore, we will not proceed according to normative rules (being, at least in part, subject to the restrictions of our own descriptive generalizations). Thus, what will be attributed will always be, in some sense, our own view, not that proscribed by our theoretical understanding. 
Gadamer suggests that we approach a text with an "anticipation of perfection." At first this may sound like an unacceptable strong version of charity, until, that is, we couple this notion with the distinctions just made. We anticipate perfect agreement between the text or the natives and ourselves, not agreement between them and normative rationality. I think in terms of the sun rising and setting, although I realize that this is, scientifically, an inaccurate description. Still, upon meeting a native who informally spoke in terms of the earth orbiting the sun, I would be surprised. My surprise wouldn't necessarily be a response to the native's cosmological knowledge, but more likely a reaction to unusual everyday conceptualizations of the world. Of course, if my way of thinking is unusually close to logical norms, deviance from this way of thinking will surprise me. Ergo, I may be dumbfounded at the errors committed by students of elementary logic, even to the point of being unable to explain to them the basic rules of reasoning I take to be obvious. 10

Gadamer and Henderson both see initial charitable contribution, albeit weighted differently, as contributing to the future possibility of genuine differentiation between the interpreter's and the subject's beliefs. Thus, charity ends at a certain stage: for Henderson, when enough information has been gleaned to allow opinions we find inexplicable to surface; and for Gadamer, when the information our reading has provided finally proves irreconcilable with our own pre-opinions. On Henderson's view, charity is in the service of making the other's behavior explicable. We want to understand the relations of the other's beliefs and actions. Gadamer sees interpretation as directed toward agreement, with initial pre-opinions allowing disagreements to become explicit. These are then either explicated, in much the same way as Henderson describes (with the difference that they are related to our own opinions rather than necessarily scientific explanation), or resolved into agreement by our taking on the vicws of the text.

The emphasis on explicability rather than agreement follows from the sort of cases with which Henderson is concerned. Approaching other cultures from the standpoint of a technological society with advanced and articulated vicws about the world, most of our anthropological interpretation will be directed toward an understanding of why other cultures don't share our opinions. In most cases, we will have good reasons to belicve our scientific views are better. Hence, most disagrecments will be resolved by one side of the course Gadamer describes: understanding why we cannot agree with the text but must instead recognize the biases and prejudices within its tradition.

10 To be fair to Henderson, it seems unlikely that someone who reasoned according to logical norms wouldn't come to recognize that others usually don't. Thus, descriptive generalizations are born. But it seems that this generalization will be aimed at explaining why others reason differently than myself, not vice versa though I, as the perfect reasoner, would be the anomaly! 
But the Principle of Explicability does less justice to cases where we may actually become convinced that our subject is correct, and our own theories about the world are not. Again, this is due to the subjects on which Gadamer focuses. Most learning fits into this category. Consider, for instance, a student of philosophy reading Quine's Word \& Object or Heidegger's Being and Time. Depending on the student's background, he or she may in fact spend some energy explicating the faults of the world-view within these texts; on the other hand, the second of Gadamer's directions will most likely come into play in most cases. The student's own preopinions about the world will be brought into question, through the conflict of these prejudices with the view of the text. The text then makes its case, and the student may learn, i.e. come to understand the matter under discussion into a new way. For Gadamer, this latter confrontation of opinions is the more primary sort of end for an interpretive path to take. While questions of primacy are not easily answered, we may at least pose the following question: would the most appropriate codification of actual interpretive practice see "explicability" in terms of explaining the differences of the subject from the expectations of scientific descriptive generalizations, or in terms of understanding the subject's view on the matter at hand in relation to my own?

Henderson's view that, in the later stages of interpretation, one strives to model the behaviors of the subject in terms of "relevance relations" seems parallel to Gadamer's desire to "see from the other's point of view," although Henderson has perhaps articulated this goal more thoroughly. Also, it is clear that Henderson's notion of "global theory" coming into play in the interpretive process translates closely into Gadamer's notion that the subject's claims about the world are understood "in relation to the whole of one's own opinions." Gadamer is silent on the issue of methodological separatism, and the direction in which his theory points isn't immediately obvious, but he does suggest that all understanding has a hermeneutic character. Presumably, this would include understanding in the natural sciences, although what the structure of the appropriate hermencutic approach would be in such a case is not spelled out here. 11

In conclusion, the two accounts, Henderson's drawing upon recent Anglo-American work and Gadamer's upon the Continental tradition, attribute a similar structure to interpretive practice. As we have seen, each recognizes the necessity of approaching the subject to be interpreted in an initially charitable manner, this charitable attribution making way for the recognition of differences between the interpreter's expectations and the

11 It seems that Gadamer would be more inclined to group the natural sciences under interpretation than vice versa. The reasons for this have to do with his theory concerning Wirkungsgeschicht ("the history of influence"), not explicitly discussed here: "Understanding is a process in the history of intluence, and it could be proven that it is in the linguisticality belonging to all understanding that the hermeneutical event makes its path" (p. 78). See also the editors' introduction, p. 33. 
subject's revelations. In this regard, the two accounts are closer to each other than to predecessors in each's respective tradition in many cases. Possibly due to differences of focus, however, the theories diverge with respect to the weighting applied to the expectations of the interpreter, and in what is regarded as the ultimate goal of interpretation. The relationship between these approaches, and the questions raised by their differences, may indicate the direction new work in this area could take. Among the questions which need to be addressed are the interpreter's own relation to the theories about the world which are applied charitably in the early stages of interpretation, and whether the Principle of Explicability provides a satisfactorily encompassing view of interpretation in the case where differences are resolved in favor of the subject's view of the world rather than the interpreter's. 